\title{
From Damage Percolation to Crack Nucleation Through Finite Size Criticality
}

\author{
Ashivni Shekhawat, ${ }^{1}$ Stefano Zapperi, ${ }^{2,3}$ and James P. Sethna ${ }^{1}$ \\ ${ }^{1}$ LASSP, Physics Department, Clark Hall, Cornell University, Ithaca, New York 14853-2501, USA \\ ${ }^{2}$ Consiglio Nazionale delle Ricerche-IENI, Via R. Cozzi 53, 20125 Milano, Italy \\ ${ }^{3}$ ISI Foundation, Via Alassio 11C, 10126 Torino, Italy \\ (Received 6 October 2012; published 29 April 2013)
}

\begin{abstract}
We present a unified theory of fracture in disordered brittle media that reconciles apparently conflicting results reported in the literature. Our renormalization group based approach yields a phase diagram in which the percolation fixed point, expected for infinite disorder, is unstable for finite disorder and flows to a zero-disorder nucleation-type fixed point, thus showing that fracture has a mixed first order and continuous character. In a region of intermediate disorder and finite system sizes, we predict a crossover with mean-field avalanche scaling. We discuss intriguing connections to other phenomena where critical scaling is only observed in finite size systems and disappears in the thermodynamic limit.
\end{abstract}

Brittle fracture in disordered media intertwines two phenomena that seldom coexist, namely, nucleation and critical fluctuations. The usual dichotomy of thought between nucleated and continuous transitions makes the study of fracture interesting. Even more intriguing is the fact that crack nucleation happens at zero stress in the thermodynamic limit: smaller is stronger and larger is weaker. This makes the existence of critical fluctuations in the form of clusters and avalanches of all sizes even more mysterious. What kind of critical point governs a phase transition that happens at zero applied field (stress) in the thermodynamic limit, and what is the universality class of such a transition? How do self-similar clusters, extremely rough crack surfaces, and scale invariant avalanches ultimately give rise to sharp cracks and localized growth? These questions have been addressed previously via a host of different theories, such as those based on percolation and multifractals [1-4], spinodal modes and meanfield criticality [5], and classical nucleation [6-9]. In this Letter, we present a theoretical framework based on the renormalization group and crossover scaling that unifies the seemingly disparate descriptions of fracture into one consistent framework.

Fracture in disordered media is the result of a complex interplay between quenched heterogeneities and long-range stress fields leading to diffuse damage throughout the sample, and local stress concentration favoring the formation of sharp localized cracks. The self-affine morphology of cracks [10], the power-law statistics of avalanche precursors [11-14], and the scale dependence of the failure strength distribution [15-17] all result from this competition. Disordered fracture can be understood in the limit of infinitesimal as well as infinite disorder. Infinitesimal disorder means a perfect crystalline material with just a few isolated defects (say a missing atom or a microcrack). In this limit, fracture statistics can be understood as a nucleation-type first order phase transition [6-9]. In the limit of infinite disorder, stress concentration becomes irrelevant and fracture progresses via uncorrelated percolationlike damage $[1,2]$. This mapping to percolation theory becomes rigorously valid when the disorder distribution is not normalizable (or very broad, in the language of multifractals) [1]. The situation is more interesting at intermediate disorder, where unlike typical first order transitions, crack nucleation is preceded by avalanches with powerlaw distributions and mean-field exponents [5,18-20], sometimes interpreted as a signature of a spinodal point [5]. Our renormalization group based theory unifies the above descriptions into a single phase diagram.

We use a two-dimensional fuse network to model disordered brittle materials. A description of the disordered fuse network model that we study can be found in any number of references $[2-5,15,21,22]$. Briefly, we consider a periodic network of fuses arranged in a square lattice of size $L$ tilted by $45^{\circ}$ (the so-called 'diamond lattice') [see Fig. 1(a)]. Each fuse is assigned a quenched current threshold from a common distribution with a cumulative distribution function $F(\cdot)$. If the current through a fuse exceeds its threshold, then the fuse is burned and is removed from the network; i.e., its conductance is set to zero. The current through the network is ramped quasistatically, and fuses are burned one at a time until the network becomes nonconducting, at which point the network is said to be fractured. We assign thresholds between 0 and 1 , specifically we take $F(x)=x^{\beta}, \beta>0$. This form of distribution of thresholds serves as a model for a generic distribution with a power-law tail at the origin, and has been studied widely [2-5]. In this model the limit $\beta \rightarrow 0$ corresponds to infinite disorder, while the limit $\beta \rightarrow \infty$ corresponds to infinitesimal disorder. Figure 1 shows a schematic of an undamaged fuse network [see Fig. 1(a)], and realizations of fractured networks for various values of the parameter $\beta$. Notice how the damage looks percolationlike for small $\beta$ while a single crack appears for large $\beta$. 


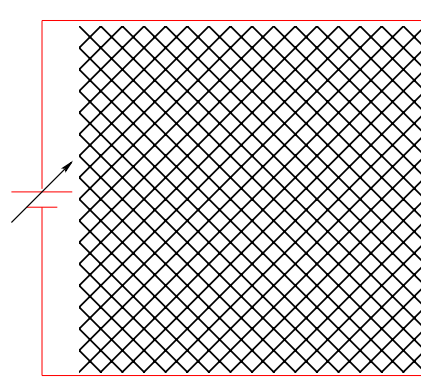

(a) A fuse network

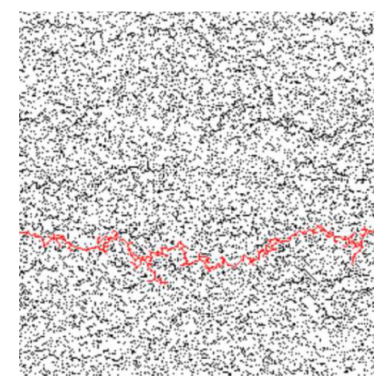

(c) $\beta=0.5$

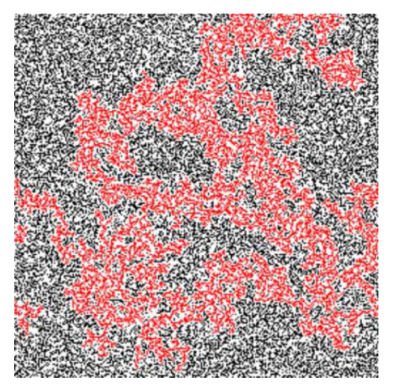

(b) $\beta=0.03$

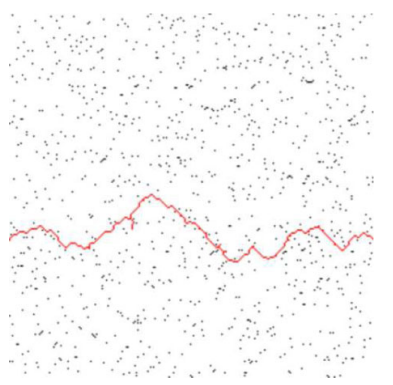

(d) $\beta=3.0$
FIG. 1 (color online). Fuse network model. (a) Schematic of a fuse network. Periodic boundary conditions are used in the horizontal direction. (b)-(d) Fractured sample for various values of the parameter $\beta$; the spanning cluster (or crack) is colored red. There is a smooth crossover from percolationlike behavior for small $\beta$ to nucleated cracks at large $\beta$.

We begin by arguing that crack-tip stress concentration is a relevant perturbation to the infinite disorder percolation critical point. Our assertion implies that percolationlike behavior is a finite-size crossover effect. This is consistent with recent results showing that even an arbitrarily small cutoff in the threshold distribution (at $\beta=0$ ) leads to a crossover away from percolation at large system sizes [23]. We calculate self-consistent upper and lower bounds for the stress and damage fraction at failure, and show that all of these quantities vanish in the limit of large $L$. This will establish that percolation cannot be the dominant behavior for large $L$, since percolation demands that the damage fraction be finite. Let $\sigma_{f}, \phi_{f}$ be the stress and the damage fraction at failure, respectively. The lower bound on both quantities is trivially equal to 0 . The upper bound is obtained self-consistently. Let us assume that $\phi_{f}<\phi_{f}^{+} \ll$ 1 , where $\phi_{f}^{+}$is an upper bound on $\phi_{f}$, and similarly $\sigma_{f}<$ $\sigma_{f}^{+} \ll 1$. Let, if possible, the damage be percolationlike, so that $\phi_{f}^{+}=F\left(\sigma_{f}^{+}\right)$[24]. The stress at the tip of a crack of length $l$ (lattice units) is given by $\sigma_{\text {tip }}(l) \approx \sigma_{f}^{+}(1+\alpha \sqrt{l})$, where $\alpha$ is a lattice dependent constant. Thus, the length of a critical crack at a given stress and damage fraction is $l_{c r}\left(\sigma_{f}^{+}\right) \sim 1 /\left(\sigma_{f}^{+}\right)^{2} \alpha^{2}+$ h.o.t. The probability that a critical crack forms at a given lattice site is at least $F\left(\sigma_{f}^{+}\right)^{l_{c r}}$. Since there are $L^{2}$ sites in the lattice, the probability of one such crack appearing on the entire lattice is at least
$L^{2} F\left(\sigma_{f}^{+}\right)^{l_{c r}}$ [6]. At the failure stress this probability is 1; thus, $\sigma_{f}^{+}$can be obtained by solving $L^{2} F\left(\sigma_{f}^{+}\right)^{l_{c r}\left(\sigma_{f}^{+}\right)}=1$. It can be proved that the solution $\sigma_{f}^{+}(L) \rightarrow 0$ as $L \rightarrow \infty$; thus, $\phi_{f}^{+}=F\left(\sigma_{f}^{+}\right) \rightarrow 0$.

We have established that percolation is unstable to nucleation; however, the crossover length is expected to be rather large. The reason for this effect is that $\phi_{f}^{+}(L)$ decays very slowly with $L$. This slow decay is expected to also manifest itself in the form of large finite size effects. The rate of decay obviously depends on $F(\cdot)$; for $F(x)=x^{\beta}$ one can show that $\phi_{f}^{+}(L) \sim(\beta / 2 \log L)^{\beta / 2}$. More sophisticated estimates that account for stress concentration during the growth of the critical crack, as opposed to percolationlike growth assumed here, yield similar results. The convergence becomes extremely slow as $\beta$ approaches 0 , meaning that the percolation threshold will be reached before nucleation of the critical crack for increasing larger system sizes. This is consistent with the previous studies that found that the fuse network can be mapped onto a percolation problem in the limit of $\beta \rightarrow 0$ [2]. However, one should note the subtle point that order of limits matters since percolation is ultimately unstable to nucleation at any $\beta$.

The avalanche behavior associated with fracture can be understood via a simple argument. The argument is valid in the vicinity of the critical point and breaks down for very large $L$. Consider an avalanche that starts with a bond breaking at a stress $\sigma(\ll 1)$ and damage fraction $\phi(\ll 1)$. Linear elasticity predicts that the change in the stress field due to the breaking of the bond $c(r, \sigma)$ decays as $c(r, \sigma) \sim$ $\sigma / r^{2}$ (ignoring the dipolar directional dependence), where $r$ is the distance from the broken bond. The probability that a bond at distance $r$ breaks in response to this change in stress is approximately given by $F^{\prime}(\sigma) c(r, \sigma)$. Thus, the expected number of bonds that break in response to stress change due to one bond breaking is given by $\lambda \sim$ $\int_{1}^{L} r d r F^{\prime}(\sigma) c(r, \sigma) \sim F^{\prime}(\sigma) \sigma \log L$. Substituting the form $F(x)=x^{\beta}$ gives $\lambda(\sigma, \beta, L) \sim \beta \sigma^{\beta} \log L=\beta \phi \log L$. This shows that $\lim _{\beta \rightarrow 0} \lambda(\beta, \phi, L)=0$ (for fixed $L$ ); thus, there are no avalanches for small $\beta$, and the damage is percolationlike. For suitable $\beta$ the avalanche progresses as a branching process, where the breaking of one bond triggers a few more and so on ( $\lambda$ is also known as the branching ratio). It is well known that the integrated avalanche size distribution for such processes is a power law with exponent $\tau_{a}=3 / 2+1=5 / 2$; for suitably large $L$ we expect the avalanche size distribution to be a power law with exponents consistent with the mean-field value of $5 / 2$ [5]. Finally, for very large $L$ (or $\beta$ ), the system flows away from the critical point and the avalanches get cut off due to nucleation effects.

All the ideas discussed so far can be encapsulated neatly in the form of crossover scaling functions. The scaling form for the cluster size distribution can be derived by using ideas of scale invariance. Let $G\left(z_{1}, \ldots, z_{n}\right)$ be a 
scale invariant function, then by definition $G(\cdot)$ should remain invariant under a rescaling by a factor $b$, i.e., $G\left(z_{1}, \ldots, z_{n}\right)=b^{\alpha_{0}} G\left(z_{1} b^{\alpha_{1}}, \ldots, z_{n} b^{\alpha_{n}}\right)$ for some constants $\alpha_{i}$. Taking $b=1+\epsilon$ and solving up to first order in $\epsilon$ gives the general form of a scale invariant function as $G\left(z_{1}, \ldots, z_{n}\right)=z_{1}^{-\alpha_{0} / \alpha_{1}} G\left(z_{2} z_{1}^{-\alpha_{2} / \alpha_{1}}, \ldots, z_{n} z_{1}^{-\alpha_{n} / \alpha_{1}}\right)$, where the universal scaling function $G(\cdot)$ and the critical exponents $\alpha_{i} / \alpha_{1}$ are characteristic of the critical point $[25,26]$. The variables $z_{i}$ represent directions in parameter space near the critical point. The directions with $\alpha_{i}>0$ belong to the relevant parameters and those with $\alpha_{i}<0$ to irrelevant parameters. We treat $\beta, 1 / L$ to be relevant parameters, and let $u$ be the leading irrelevant parameter (the largest of the negative $\alpha_{i}$ ). Thus, ignoring all irrelevant variables but the leading one, the scale invariant distribution of cluster sizes can be written as

$$
P_{c}(s \mid \beta, L)=s^{-\tau_{c}} \mathcal{F}_{c}\left(\beta L^{1 / \nu_{f}}, s L^{-1 / \sigma_{c} \nu_{f}}, u L^{-\Delta_{f} / \nu_{f}}\right) .
$$

where the subscript $c$ denotes variables associated with the clusters. We use the subscript $f$ (for fracture) to distinguish the critical exponents from their counterparts in percolation theory. We know that in the limit of $\beta \rightarrow 0$ (at fixed $L$ ) the cluster size distribution should reduce to the distri bution of percolation clusters at the critical point; thus, we can deduce three critical exponent combinations, namely $\tau_{c}=187 / 91=2.0549, \quad \sigma_{c} \nu_{f}=48 / 91=0.5275$, and $\Delta_{f} / \nu_{f}=72 / 48=1.5$ [27]. Even though the clusters created in fracture are loopless [23], the static properties of loopless percolation are identical to usual percolation; thus, the use of percolation critical exponents is valid [28]. The moments of the cluster size distribution should scale as (taking a Taylor expansion in $u L^{-\Delta_{f} / \nu_{f}}$ for large $L$ )

$$
\begin{aligned}
\left\langle s_{c}^{n}\right\rangle= & L^{\left(n+1-\tau_{c}\right) / \sigma_{c} \nu_{f}}\left(\mathcal{J}_{n}^{c}\left(\beta L^{1 / \nu_{f}}\right)\right. \\
& \left.+L^{-\Delta_{f} / \nu_{f}} \mathcal{K}_{n}^{c}\left(\beta L^{1 / \nu_{f}}\right)\right),
\end{aligned}
$$

where $\mathcal{J}_{n}^{c}(\cdot), \mathcal{K}_{n}^{c}(\cdot), n=2,3, \ldots$ are universal scaling functions [29]. From a data fitting perspective, it is easier to deal with the moments (as opposed to the distribution function) because $\mathcal{J}_{n}^{c}(\cdot), \mathcal{K}_{n}^{c}(\cdot)$ are functions of just one scaling variable. The functions for the avalanche size distribution are completely analogous,

$$
\begin{aligned}
P_{a}(s \mid \beta, L)= & s^{-\tau_{a}} \mathcal{F}_{a}\left(\beta L^{1 / \nu_{f}}, s L^{-1 / \sigma_{a} \nu_{f}}, u L^{-\Delta_{f} / \nu_{f}}\right), \\
\left\langle s_{a}^{n}\right\rangle= & L^{\left(n+1-\tau_{a}\right) / \sigma_{a} \nu_{f}}\left(\mathcal{J}_{n}^{a}\left(\beta L^{1 / \nu_{f}}\right)\right. \\
& \left.+L^{-\Delta_{f} / \nu_{f}} \mathcal{K}_{n}^{a}\left(\beta L^{1 / \nu_{f}}\right)\right),
\end{aligned}
$$

where $\tau_{a}$ is expected to be close to its mean-field value of $5 / 2$.

We have done numerical simulations to verify our theoretical predictions. We did extensive statistical sampling of systems of size up to $L=128$ and $\beta$ between 0.03 and 8 . In order to fit the data to the scaling predictions we use the following functional forms for the scaling functions for the moments of the cluster size distribution [with $y_{c}(x) \equiv\left(\log x-\mu_{c}\right) / \alpha_{c}$ ]

$$
\begin{aligned}
& \mathcal{J}_{n}^{c}(x)=a_{0, n} \operatorname{erf}\left(y_{c}(x)\right)+e^{-\left(y_{c}(x)\right)^{2}} \sum_{i=0}^{i=m} A_{i, n}^{c} H_{i}\left(y_{c}(x)\right), \\
& \mathcal{K}_{n}^{c}(x)=a_{1, n} \operatorname{erf}\left(y_{c}(x)\right)+e^{-\left(y_{c}(x)\right)^{2}} \sum_{i=0}^{i=m} B_{i, n}^{c} H_{i}\left(y_{c}(x)\right),
\end{aligned}
$$

where $\mu_{c}, \alpha_{c}, a_{0, n}, a_{1, n}, A_{i, n}^{c}, B_{i, n}^{c}$ are fitting parameters, $\operatorname{erf}(\cdot)$ is the error function, and $H_{i}(\cdot)$ is the $i$ th Hermite polynomial. We use the first three Hermite polynomials in the expansion, i.e., $m=3$. The corresponding forms for the avalanches are [with $y_{a}(x) \equiv\left(\log x-\mu_{a}\right) / \alpha_{a}$ ]

$$
\begin{gathered}
\mathcal{J}_{n}^{a}(x)=e^{-\left(y_{a}(x)\right)^{2}} \sum_{i=0}^{i=m} A_{i, n}^{a} H_{i}\left(y_{a}(x)\right), \\
\mathcal{K}_{n}^{a}(x)=e^{-\left(y_{a}(x)\right)^{2}} \sum_{i=0}^{i=m} B_{i, n}^{a} H_{i}\left(y_{a}(x)\right) .
\end{gathered}
$$

The forms of the scaling functions are chosen so that they have the correct asymptotic behavior. As discussed previously, we know that $\lim _{\beta L^{1 / v_{f} \rightarrow 0, \infty}} \mathcal{J}_{n}^{a}\left(\beta L^{1 / \nu_{f}}\right)=0$ since there are no avalanches for very small $\beta$ (at fixed $L$ ) and at very large $L$ (at fixed $\beta$ ). On the other hand we know $\lim _{\beta L^{1 / \nu_{f} \rightarrow 0}} \mathcal{J}_{n}^{c}\left(\beta L^{1 / \nu_{f}}\right)=C$ for some constant $C$ (according to percolation theory) and $\lim _{\beta L^{1 / \nu_{f} \rightarrow \infty}} \mathcal{J}_{n}^{c}\left(\beta L^{1 / \nu_{f}}\right)=0$ since there are no clusters in

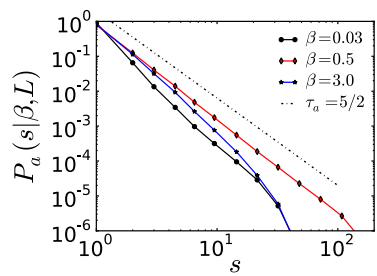

(a) Avalanche size distribution

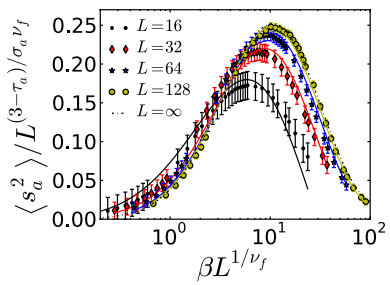

(c) Scaling collapse of $\left\langle s_{a}^{2}\right\rangle$

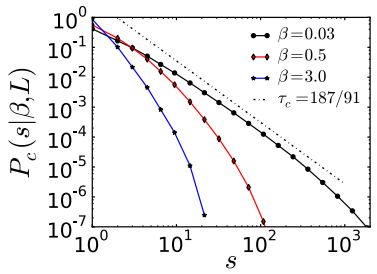

(b) Cluster size distribution

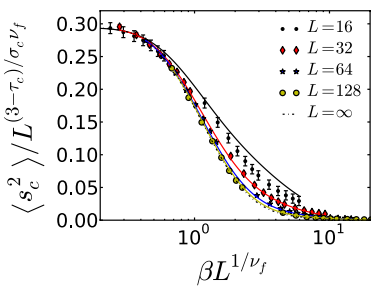

(d) Scaling collapse of $\left\langle s_{c}^{2}\right\rangle$
FIG. 2 (color online). Scaling theory of fracture. (a) The avalanche size distribution shows a power law consistent with the mean-field exponent of $5 / 2$ for moderate $\beta(=0.5$ at $L=128)$. As expected, the power law is distorted for much smaller or larger $\beta$. (b). The cluster size distribution shows a power law that is consistent with the exponent predicted by percolation theory $(=187 / 91)$. The power-law cutoff becomes smaller as one moves away from the critical point. (c), (d). The scaling forms fit the data well, confirming the predictions of the scaling theory. Higher moments of the distributions fit the scaling forms as well (not shown here). Notice the significant finite-size effects as the data get closer to the $L=\infty$ curve with increasing system sizes. 


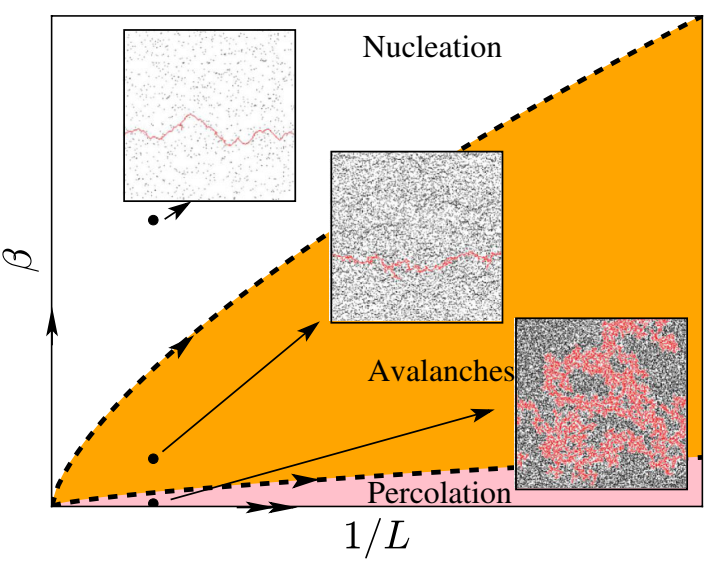

FIG. 3 (color online). Phase diagram for brittle fracture in disordered media [4]. Disorder decreases along the $\beta$ axis; nucleation governs the behavior for small disorder or long length scales. Percolation is characteristic of the large disorder regime, while the crossover region exhibits interesting critical behavior in the form of scale free distributions of avalanche sizes. The topology of the fractured samples evolves from percolationlike damage for large disorder to well defined sharp cracks in the nucleated regime. The phase boundaries are quantitatively somewhat arbitrary, and are set at the value of the scaling variable $\beta L^{1 / \nu_{f}}$ at which the second moment of the avalanche size become half of its peak value (for the avalanche phase); the boundary of the percolation phase is found analogously.

the nucleation dominated regime away from the critical point. The forms used here satisfy all these requirements.

Figure 2 shows the size distributions as well as fits to the scaling forms. It is evident that the data are consistent with the scaling theory. Based on joint fits for $n=2,3(n=3$ is not shown in Fig. 2), we estimate the following values of the critical exponents: $\nu_{f}=1.56 \pm 0.30, \sigma_{a}=0.47 \pm$ $0.15, \Delta_{f}=2.35 \pm 1.50, \sigma_{c}=0.34 \pm 0.08$. For the fits shown in Fig. 2 the exponent $\tau_{a}$ is held at its mean-field value of $5 / 2$, while unbiased fits yield $\tau_{a}=2.45 \pm 0.25$. The scaling exponent $\tau_{c}$ and the exponent combinations $\sigma_{c} \nu_{f}, \Delta_{f} / \nu_{f}$ are held at their theoretical values of $187 / 91$ and 48/91, 72/48, respectively. The statistical error bars are much smaller than the error bars reported here. We have estimated the error bars due to systematic errors by using a variety of techniques such as varying the number of terms in the scaling functions, trying different fitting forms, varying the critical range for the fits, varying the error bars on the data over a reasonable range, etc. Figure 3 shows the phase diagram that emerges from our analysis [30]. In the $\beta-1 / L$ space, curves along which the scaling variable $\beta L^{1 / \nu_{f}}$ attains a critical value demarcate the boundary between qualitatively different behavior. Note that the exact position of the boundaries is somewhat arbitrary, since this is a not an abrupt (first order) transition; however, the diagram is qualitatively accurate.

The critical phenomena associated with fracture has several intriguing characteristics. First, the scaling function associated with the avalanches has a singularity at $0\left[\lim _{\beta L^{1 / \nu_{f} \rightarrow 0}} \mathcal{J}_{n}^{a}\left(\beta L^{1 / \nu_{f}}\right)=0\right]$ that subdues the avalanche behavior as the critical point is approached. Second, there is no point in the phase diagram (except for the $\beta=0$ limit) that shows any critical phenomena in the limit of $L \rightarrow \infty$. Thus, scale invariance itself becomes a finite-size effect; perhaps this phenomena should be named finitesized criticality. Finally, it is rather remarkable that the critical phenomena (typically associated with continuous phase transitions) gives way to nucleation (a first order transition) in the limit of long length scales. Thus, fracture has mixed first order and continuous transition character. Transitions of mixed first order and continuous character have become something of a theme in the past decade or so. Recently we noted that the Mott transition and dielectric breakdown have a mixed character [31]; similar findings have been reported in a variety of fields such as jamming transitions, rigidity percolation [32], and phase-separated manganites [33].

In conclusion, we have presented a scaling theory of fracture that builds on renormalization group ideas and unifies several disparate results in the field. Our theory shows that percolationlike behavior as well as the scale invariant precursor avalanches leading to fracture are finitesize effects. We show that on long length scales brittle fracture is always nucleated. We hope that our analysis will pave the way for a deeper understanding of the many mysteries associated with the phenomenon of fracture.

We would like to thank S.L. Phoenix for insightful discussions. A. S. and J.P. S. were supported by the DOE under Grant No. DOE-BES DE-FG02-07ER46393. S.Z. acknowledges financial support from ERC-AdG-2011 SIZEFFECTS. This research was supported in part by the National Science Foundation through TeraGrid resources provided by the Louisiana Optical Network Initiative (LONI) under Grant No. TG-DMR100025.

[1] S. Roux, A. Hansen, H. Herrmann, and E. Guyon, J. Stat. Phys. 52, 237 (1988).

[2] A. Hansen and J. Schmittbuhl, Phys. Rev. Lett. 90, 045504 (2003).

[3] A. Hansen, E. L. Hinrichsen, and S. Roux, Phys. Rev. B 43, 665 (1991).

[4] R. Toussaint and A. Hansen, Phys. Rev. E 73, 046103 (2006).

[5] S. Zapperi, P. Ray, H. E. Stanley, and A. Vespignani, Phys. Rev. Lett. 78, 1408 (1997).

[6] P. M. Duxbury, P. L. Leath, and P. D. Beale, Phys. Rev. B 36, 367 (1987).

[7] P. M. Duxbury, P.D. Beale, and P. L. Leath, Phys. Rev. Lett. 57, 1052 (1986).

[8] C. Manzato, A. Shekhawat, P. K. V. V. Nukala, M. J. Alava, J. P. Sethna, and S. Zapperi, Phys. Rev. Lett. 108, 065504 (2012). 
[9] B. K. Chakrabarti and L. G. Benguigui, Statistical Physics of Fracture and Breakdown in Disordered Systems (Oxford University, New York, 1997).

[10] D. Bonamy and E. Bouchaud, Phys. Rep. 498, 1 (2011).

[11] P. C. Hemmer and A. Hansen, J. Appl. Mech. 59, 909 (1992).

[12] A. Petri, G. Paparo, A. Vespignani, A. Alippi, and M. Costantini, Phys. Rev. Lett. 73, 3423 (1994).

[13] A. Garcimartin, A. Guarino, L. Bellon, and S. Ciliberto, Phys. Rev. Lett. 79, 3202 (1997).

[14] L. I. Salminen, A. I. Tolvanen, and M. J. Alava, Phys. Rev. Lett. 89, 185503 (2002).

[15] M. J. Alava, P. K. V. V. Nukala, and S. Zapperi, Adv. Phys. 55, 349 (2006).

[16] Z.P. Bazant, Proc. Natl. Acad. Sci. U.S.A. 101, 13400 (2004).

[17] D. G. Harlow and S. L. Phoenix, J. Compos. Mater. 12, 195 (1978).

[18] A. Hansen and P.C. Hemmer, Phys. Lett. A 184, 394 (1994).

[19] S. Zapperi, P. K. V. V. Nukala, and S. Simunovic, Phys. Rev. E 71, 026106 (2005).

[20] S. Zapperi, P. K. V. V. Nukala, and S. Simunovic, Physica (Amsterdam) 357A, 129 (2005).

[21] P. K. V. V. Nukala and S. Simunovic, J. Phys. A 36, 11403 (2003).

[22] B. Kahng, G. G. Batrouni, S. Redner, L. de Arcangelis, and H. J. Herrmann, Phys. Rev. B 37, 7625 (1988).
[23] A. A. Moreira, C. L. N. Oliveira, A. Hansen, N. A. M. Araujo, H. J. Herrmann, and J. J. S. Andrade, Phys. Rev. Lett. 109, 255701 (2012).

[24] A calculation based on effective medium theory yields $\phi=F\left(\sigma /\left(1-\phi^{2}\right)\right) \sim F(\sigma)+\mathcal{O}\left(\sigma \phi^{2}\right)$. We ignore the higher order terms.

[25] J.P. Sethna, K. A. Dahmen, and C. R. Myers, Nature (London) 410, 242 (2001).

[26] Other orderings of variables are equally valid, such as $G\left(z_{1}, \ldots, z_{n}\right)=z_{2}^{-\alpha_{0} / \alpha_{2}} G_{2}\left(z_{1} z_{2}^{-\alpha_{1} / \alpha_{2}}, \ldots, z_{n} z_{2}^{-\alpha_{n} / \alpha_{2}}\right)$, etc. See Ref. [25] for details.

[27] R. M. Ziff, Phys. Rev. E 83, 020107 (2011).

[28] F. Tzschichholz, A. Bunde, and S. Havlin, Phys. Rev. A 39, 5470 (1989).

[29] This scaling relation is valid only if $n+1-\tau_{c}>0$; we find $\tau_{c}=187 / 91$, thus, $n \geq 2$.

[30] Reference [4] has foreshadowed our 'phase diagram' of Fig. 3, with their 'diffuse phase' (see Fig. 4 of Ref. [4]) corresponding to our avalanche regime, but their results were not based a scaling description. Indeed, our crossover analysis is inconsistent with their phase boundary (or any phase boundary as $1 / L \rightarrow 0$ ).

[31] A. Shekhawat, S. Papanikolaou, S. Zapperi, and J.P. Sethna, Phys. Rev. Lett. 107, 276401 (2011).

[32] W. G. Ellenbroek and X. Mao, Europhys. Lett. 96, 54002 (2011).

[33] L. Ghivelder, R. S. Freitas, M. G. das Virgens, M. A. Continentino, H. Martinho, L. Granja, M. Quintero, G. Leyva, P. Levy, and F. Parisi, Phys. Rev. B 69, 214414 (2004). 